\title{
El efecto antisenescente del resveratrol reduce la tasa de ablandamiento poscosecha de chirimoya
}

\section{The anti-senescence effect of resveratrol reduces postharvest softening rate in cherimoya fruit}

\author{
Aaran Aquilino Morales Pérez ${ }^{1}$, Omar Franco-Mora, 2, Álvaro Castañeda- \\ Vildozola $^{2}$, Edgar Jesús Morales-Rosales ${ }^{2}$ \\ ${ }^{l}$ Programa doctoral en Ciencias Agropecuarias y Recursos Naturales. Universidad Autónoma del Estado de México, \\ Campus Universitario El Cerrillo Piedras Blancas. C. P. 50000, Toluca, Estado de México. \\ 2 Laboratorio de horticultura, Facultad de Ciencias Agrícolas, Centro de Investigación y Estudios Avanzados en \\ Fitomejoramiento, Universidad Autónoma del Estado de México, Campus Universitario El Cerrillo Piedras Blancas. C. \\ P. 50000, Toluca, Estado de México.
}

Recibido 05 febrero 2014. Aceptado 18 marzo 2014.

\begin{abstract}
Resumen
El fruto de chirimoya (Annona cherimola Mill.) es muy susceptible al deterioro poscosecha debido a su naturaleza climatérica. Con el fin de observar el efecto antisenescente del resveratrol (RVS), éste bioregulador vegetal se aplicó en frutos de 'Fino de Jete' y 'Bronceada' a 1,6; 0,16; 0,016 y 0 mM a los 0, 8 y 15 días antes de la cosecha (DAC). A los 1, 7 y 15 días después de la cosecha (DDC) se analizaron variables bioquímicas y biofísicas. Al termino de 15 días de almacenamiento a temperatura ambiente, en relación al control, 1,6 mM RVS, aplicado 15 DAC, disminuyó el ablandamiento del fruto $78 \%$ para chirimoya 'Fino de Jete' y $54 \%$ para 'Bronceada'. A los 15 DDC se realizó la evaluación sensorial a frutos tratados 8 y 15 DAC, los resultados mostraron que los frutos de mayor aceptación fueron los tratados con 1,6 mM RVS ya sea a los 8 y 15 DAC, al ser calificados como de mejor aspecto, aroma y sabor.

Palabras clave: Ablandamiento, Annona cherimola Mill., climatérico, deshidratación, evaluación sensorial.
\end{abstract}

\begin{abstract}
Due to its climateric behavior, the cherimoya fruit (Annona cherimola Mill.) is a very perishable commodity. Present research aimed to observe the anti-senescence effect of resveratrol (RVS); this plant bioregulator was applied at 1.6, 0.16, 0.016 and $0 \mathrm{mM}$ at 0,8 and 15 days before harvest (DBH). At 1,7 and 15 days of postharvest life, several physical and biochemical determinations were performed on the cherimoya fruit. After 15 days under room temperature conditions, in relation to control fruit, those fruit with $1.6 \mathrm{mM}$ RVS applied 15 DBH reduced skin softening rate $78 \%$ and $54 \%$ for 'Fino de Jete' and 'Bronceada', respectively. Similarly, after 15 days of storage, a non-trained group of tasters preferred those fruit treated with $1.6 \mathrm{mM}$ RVS 15 DBH; they qualified the fruit as better than the control fruit in presentation, aroma and taste.
\end{abstract}

Keywords: Softening, Annona cherimola Mill., climateric, water loss, sensorial analysis.

\section{Introducción}

La chirimoya (Annona cherimola Mill.) es una fruta que aporta energéticos, minerales y vitaminas (Ferrucci, 1997); sin embargo la producción y consumo de esta fruta son limitados debido, en parte, a su bajo potencial de almacenamiento y transporte. El almacenamiento en fresco genera bronceado de cáscara, además el fruto es muy sensible a enfermedades fúngicas. $\mathrm{La}$ técnica más común para incrementar la vida poscosecha de esta fruta es la refrigeración (Ramos y Martínez, 1998), pero este tipo de almacenamiento también puede generar enfermedades fungosas (Cerdas et al., 2006). Además, la exposición prolongada a bajas temperaturas está limitada por su alta susceptibilidad al daño por frío (Lizana e Irarrazabal, 1984).

\footnotetext{
* Autor para correspondencia

E-mail: ofrancom@uamex.mx (O. Franco-Mora)
} 
La chirimoya inmediatamente después de la cosecha presenta una textura firme. Sin embargo, Cerdas et al. (2006) mencionan que una vez madura se vuelve muy suave, lo cual dificulta su transporte y limita su calidad. En diversas especies, el ablandamiento del fruto implica la degradación de sustancias pépticas; constituyéndose como la mayor causa de afectación poscosecha y de pérdidas económicas (Asif y Nath, 2005). En chirimoya poco se sabe sobre sus propiedades mecánicas y como son afectadas en poscosecha (Palma et al., 1993). Sin embargo, en un estudio de Zamorano et al. (1999), se indica que la firmeza de la chirimoya pasó de 230 a 40 $\mathrm{N}$ en 15 días de almacenamiento en condiciones de aire corriente. En el mismo documento se menciona que las atmósferas controladas son una buena opción para disminuir el ablandamiento de la chirimoya en poscosecha. Por ello, a la par de un mejor entendimiento de la fisiología poscosecha, se hace necesario buscar métodos para mejorar su almacenamiento, preferentemente de manera inocua para el consumidor y el ambiente.

El resveratrol (RVS) se muestra como una posible alternativa para incrementar la vida poscosecha de frutas y varias hortalizas. Jiménez et al. (2005) encontraron que la aplicación de RVS en manzana (Malus comunis L.), aguacate (Persea americana Mill.), tomate (Solanum lycopersicum), pimiento (Capsicum annuum), fresa (Fragaria $\times$ ananasa) y uva (Vitis vinifera) incrementó la calidad sensorial, referido a la textura y sabor, así como vida de almacenamiento y calidad nutricional. En mandarina (Citrus unshiu Marc.), RVS inhibió la decoloración de la cáscara; efecto atribuido a sus propiedades antioxidantes (Cherukuri et al., 2007). Además, Montero et al. (2000b) indicaron que se han encontrado beneficios antifúngicos en la aplicación exógena de RVS en la cáscara de uva. Hasta nuestro conocimiento, no hay reportes de uso de RVS en chirimoya, por consiguiente, el objetivo de esta investigación fue evaluar el efecto de RVS en la fisiología poscosecha de la fruta de chirimoya en cultivares 'Fino de Jete' y 'Bronceada', así como en la calidad de la misma.

\section{Material y métodos}

\section{Material vegetal}

Los frutos de chirimoya 'Fino de Jete' y 'Bronceada' fueron cosechados de árboles de 11 años, cultivados en un huerto experimental del Centro de Investigación Científico y Tecnológico del Estado de México (CICTAMEX) Fundación Sánchez Colín. El huerto está ubicado en Coatepec Harinas, México a $18^{\circ} 46^{\prime} 38^{\prime \prime}$ L. N., $99^{\circ}$ 46' 38' L. W., y $2240 \mathrm{~m}$ de altitud. Los frutos tratados con RVS fueron producto de polinización manual, y posteriormente seleccionados al azar de la parte basal y media del árbol. La aplicación de RVS en solución con agua destilada a dosis de 1,6; 0,$16 ; 0,016$ y $0 \mathrm{mM}$ se realizó con un pincel a 15,8 y 0 días antes de la cosecha (DAC). Una vez que los frutos fueron cosechados, se empacaron y transportaron de manera convencional al laboratorio de Horticultura de la Universidad Autónoma del Estado de México. La fruta se colocó en estantes simulando su comercialización; y se analizaron en 1, 7 y 15 días después de la cosecha (DDC).

\section{Pérdida de peso}

Los frutos se pesaron en una balanza semianalítica ELB3000, Shimadzu. El primer registro, peso de cosecha, se estableció como $100 \%$ de producto fresco. Los registros posteriores fueron transformados en porcentaje de peso, siempre, en relación con el peso inicial del fruto.

\section{Firmeza}

La firmeza de la fruta se evaluó mediante una prueba de compresión con un texturómetro Shimadzu Ez-Test equipado con una celda de carga de $50 \mathrm{~kg}$ y un embolo de superficie plana de $50 \mathrm{~mm}$ de diámetro. Bajo estas condiciones, la fruta

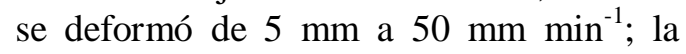


carga máxima necesaria se reportó en Newtons. Para ello, el fruto fue colocado en la plataforma bien nivelada y se realizó la lectura de firmeza en el plano ecuatorial del fruto. Después de obtener la primera lectura, se rotó el fruto a $180^{\circ}$ para obtener el segundo resultado.

Color $\left(\mathbf{L}^{*}, \mathbf{a}^{*} \mathbf{y} \mathbf{b}^{*}\right)$, cromaticidad $\mathbf{y}$ matiz

Para la determinación de color se utilizó un colorímetro Chroma CR-400, Konica, Minolta. La lente del colorímetro se colocó directamente sobre tres áreas representativas del color de la cáscara en cada fruto. Para el cálculo de cromaticidad se usó la fórmula $\mathrm{C}=\left(\mathrm{a}^{2}+\mathrm{b}^{2}\right)^{1 / 2}$ y para el ángulo de matiz Hue $=\left(\tan ^{-1} \mathrm{~b} / \mathrm{a}\right)($ Kabelka et al., 2004).

\section{Azúcares reductores}

Se pesaron $10 \mathrm{~g}$ de pulpa, la cual se maceró y se procedió a la cuantificación de azúcares reductores de acuerdo al método del Codex (CCS, 1981). Los resultados se expresaron en porcentaje de dextrosa equivalente (DE).

\section{Ácido ascórbico}

Fue determinado por yodimetría. Se pesaron $10 \mathrm{~g}$ de pulpa, se maceró y se procedió de acuerdo al método de Food Chemicals Codex (CCS, 1981). Los resultados son presentados en miligramos por kilogramo de peso fresco $\left(\mathrm{mg} \mathrm{kg}^{-1} \mathrm{PF}\right)$.

\section{Sólidos solubles totales (SST)}

Se midieron con un refractómetro manual (Atago, Japan), los resultados se muestran en grados Brix $\left({ }^{\circ} \mathrm{B}\right)$.

\section{Evaluación sensorial}

Los frutos de ambos cultivares, tratados a 8 y 15 DAC, con 1,6 mM RVS se analizaron sensorialmente a los 15 DDC. Este análisis se realizó en prueba de nivel de grado con una escala hedónica modificada de 4 puntos; "no gusta", "gusta poco", "gusta" y "gusta mucho" (Pedrero y Panborg, 1997). El número de panelista no entrenados fue de 30 y los parámetros evaluados fueron apariencia, color, olor y sabor.

\section{Análisis estadístico}

El diseño experimental fue completamente al azar, ya que los frutos para cada tratamiento se tomaron de manera aleatoria de los árboles de chirimoya disponibles. Se tuvieron cuatro factores fijos: cultivares, día de aplicación, dosis de RVS y días de almacenamiento. El número de repeticiones por tratamiento fue el mismo, 10 frutos por repetición para la cinética de peso; y 4 repeticiones, un fruto por repetición, para el resto de las variables empleadas. En su oportunidad se indicó el número de panelistas del análisis sensorial. En cada una de las variables se realizó un análisis de varianza y cuando el valor de F fue significativo, se efectuó la comparación de medias con la prueba de Tukey ( $\mathrm{p}<0,05)$; para ello se empleó el programa estadístico SAS 9.0.

\section{Resultados y discusión}

\section{Pérdida de peso}

La pérdida de peso de frutos en pos cosecha se debe, en parte, a los procesos de transpiración y respiración, ajustes osmóticos y cambios en la concentración de carbohidratos (Valero y Serrano, 2010). En la cinética de peso fresco tanto en chirimoya 'Fino de Jete' y 'Bronceada', únicamente se encontró diferencia significativa para DDC $(\mathrm{p} \leq 0,001)$ (Tabla $1)$, lo cual no es efecto de RVS, sino del paso natural de los días en almacenamiento. La anterior es contrario a lo indicado por Jiménez et al. (2005), quienes encontraron menor tasa de pérdida de peso con la aplicación de RVS en varias especies de frutos, sin encontrar efectos negativos en su contenido nutricional. Dichos autores indicaron que RVS actuó como un impermeabilizante reduciendo la presión del vapor de agua y por lo tanto mantuvo la firmeza de la fruta.

\section{Firmeza}

Para firmeza se encontraron diferencias significativas $(\mathrm{p} \leq 0,001)$ para todos $\operatorname{los}$ factores de estudio e interacciones posibles, con la excepción de Cv*DAC*RVS (Tabla 1). 


\section{Tabla 1}

Significancia de los valores de $\mathrm{F}$ del análisis de varianza para distintos factores de calidad de frutos de chirimoya 'Fino de Jete' y 'Bronceada' tratados en dos fechas precosecha con resveratrol.

\begin{tabular}{|c|c|c|c|c|c|c|c|}
\hline Fuente de variación & $\begin{array}{l}\text { Pérdida } \\
\text { de peso }\end{array}$ & Firmeza & $\begin{array}{l}\text { Cromati- } \\
\text { cidad }\end{array}$ & Matiz & $\begin{array}{l}\text { Azúcares } \\
\text { reductores }\end{array}$ & SST & $\begin{array}{c}\text { Ácido } \\
\text { ascórbico }\end{array}$ \\
\hline Cultiva r (Cv) & ns & $* * *$ & ns & ns & $* * *$ & $* * *$ & $* * *$ \\
\hline Aplicación (DAC) & ns & $* * *$ & ns & ns & $* * *$ & $* * *$ & $* * *$ \\
\hline Almacenamiento(DDC) & $* * *$ & $* * *$ & $* * *$ & $* * *$ & $* * *$ & $* * *$ & $* * *$ \\
\hline Dosis (RVS) & ns & $* * *$ & ns & ns & $* * *$ & ns & ns \\
\hline $\mathrm{CV} * \mathrm{DAC}$ & ns & $* * *$ & ns & ns & $* * *$ & $* * *$ & $* * *$ \\
\hline $\mathrm{Cv}^{*} \mathrm{DDC}$ & ns & $* * *$ & ns & ns & ns & ns & ns \\
\hline $\mathrm{CV}^{*} \mathrm{RVS}$ & ns & $* * *$ & ns & ns & ns & ns & ns \\
\hline DAC $*$ DDC & ns & $* * *$ & ns & ns & $* * *$ & $* * *$ & $* * *$ \\
\hline DDC*RVS & ns & $* * *$ & ns & $* * *$ & ns & ns & ns \\
\hline DAC*RVS & ns & $* * *$ & ns & ns & ns & $*$ & ns \\
\hline $\mathrm{CV}^{*} \mathrm{DAC} * \mathrm{DDC}$ & ns & $* * *$ & ns & ns & $* * *$ & $* * *$ & $* * *$ \\
\hline $\mathrm{CV}^{*} \mathrm{DAC} * \mathrm{RVS}$ & ns & ns & ns & ns & ns & ns & ns \\
\hline $\mathrm{CV}^{*} \mathrm{DDC} * \mathrm{RVS}$ & ns & $* *$ & ns & ns & ns & ns & ns \\
\hline DDC*DAC*RVS & ns & $* * *$ & ns & ns & ns & * & $* *$ \\
\hline $\mathrm{CV}^{*} \mathrm{DAC} * \mathrm{DDC} * \mathrm{RVS}$ & ns & $* * *$ & ns & ns & ns & ns & $*$ \\
\hline
\end{tabular}

DAC $=$ días antes de cosecha; $\mathrm{DDC}=$ días después de cosecha; $* * * * * * *$ significativo al 0,$05 ; 0,01$ y 0,001 respectivamente; ns= no significativo.

Al momento de la cosecha, los frutos presentaron un rango de firmeza entre 235 y $314 \mathrm{~N}$ y 210 a $258 \mathrm{~N}$ para 'Fino de Jete' y 'Bronceada', respectivamente (Tabla 2 y 3). Aunque no se determinó la firmeza al momento de aplicar RVS, esto por la misma naturaleza del experimento, se observó que dicha fitoalexina posiblemente afectó desde ese momento la firmeza.

\section{Tabla 2}

Firmeza en poscosecha de fruta de chirimoya 'Fino de Jete' con aplicación de resveratrol antes de cosecha

\begin{tabular}{|c|c|c|c|c|c|c|c|c|c|c|c|c|c|}
\hline \multirow{2}{*}{$\begin{array}{l}\text { Días antes } \\
\text { de cosecha }\end{array}$} & \multirow{2}{*}{$\begin{array}{c}\text { Resveratrol } \\
(\mathrm{mM})\end{array}$} & \multicolumn{12}{|c|}{$\begin{array}{l}\text { Firmeza de cáscara }(\mathrm{N}) \text { en poscosecha } \\
\text { (días después de cosecha) }\end{array}$} \\
\hline & & \multicolumn{4}{|c|}{1} & \multicolumn{4}{|c|}{7} & \multicolumn{4}{|c|}{15} \\
\hline \multirow{5}{*}{0} & 0 & 314 & \pm & 5 & $\mathrm{aA}$ & 21 & \pm & 0,7 & $\mathrm{bB}$ & 4 & \pm & 0,2 & $\mathrm{cC}$ \\
\hline & 0,016 & 298 & \pm & 3 & $\mathrm{aA}$ & 27 & \pm & 2,8 & $\mathrm{bB}$ & 6 & \pm & 0,5 & $\mathrm{cC}$ \\
\hline & 0,16 & 303 & \pm & 5 & $\mathrm{aA}$ & 46 & \pm & 0,9 & $\mathrm{aB}$ & 11 & \pm & 0,8 & $\mathrm{bC}$ \\
\hline & 1,6 & 309 & & 8 & $\mathrm{aA}$ & 52 & \pm & 2,0 & $\mathrm{aB}$ & 14 & \pm & 0,6 & $\mathrm{aC}$ \\
\hline & $\mathrm{F}$ & \multicolumn{4}{|c|}{1,4} & \multicolumn{4}{|c|}{65,1} & \multicolumn{4}{|c|}{70,4} \\
\hline \multirow{5}{*}{8} & 0 & 259 & \pm & 4 & $\mathrm{bA}$ & 14 & \pm & 0,8 & $\mathrm{bB}$ & 7 & \pm & 0,5 & $\mathrm{cB}$ \\
\hline & 0,016 & 268 & \pm & 4 & $\mathrm{bA}$ & 14 & \pm & 1,9 & $\mathrm{bB}$ & 16 & \pm & 0,3 & $\mathrm{bB}$ \\
\hline & 0,16 & 291 & \pm & 8 & $\mathrm{aA}$ & 31 & \pm & 1,3 & $\mathrm{aB}$ & 26 & \pm & 2,3 & $\mathrm{aB}$ \\
\hline & 1,6 & 235 & \pm & 5 & $\mathrm{abA}$ & 33 & \pm & 1,3 & $\mathrm{aB}$ & 30 & \pm & 1,5 & $\mathrm{aB}$ \\
\hline & $\mathrm{F}$ & \multicolumn{4}{|c|}{18,2} & \multicolumn{4}{|c|}{56,8} & \multicolumn{4}{|c|}{52,7} \\
\hline \multirow{5}{*}{15} & 0 & 297 & \pm & 5 & $\mathrm{abA}$ & 17 & \pm & 0,7 & $\mathrm{cB}$ & 7 & \pm & 0,5 & $\mathrm{~dB}$ \\
\hline & 0,016 & 288 & \pm & 3 & $\mathrm{bA}$ & 18 & \pm & 1,3 & $\mathrm{cB}$ & 18 & \pm & 1,3 & $\mathrm{cB}$ \\
\hline & 0,16 & 304 & \pm & 3 & $\mathrm{aA}$ & 26 & \pm & 0,9 & $\mathrm{bB}$ & 25 & \pm & 1,1 & $\mathrm{bB}$ \\
\hline & 1,6 & 292 & \pm & 1 & $\mathrm{abA}$ & 34 & \pm & 0,8 & $\mathrm{aB}$ & 32 & \pm & 1,9 & $\mathrm{aB}$ \\
\hline & $\mathrm{F}$ & \multicolumn{4}{|c|}{4,2} & \multicolumn{4}{|c|}{69,4} & \multicolumn{4}{|c|}{64,9} \\
\hline
\end{tabular}

Los datos son la media de cuatro repeticiones, un fruto por repetición. Literales minúsculas diferentes implican diferencias estadísticas en relación a columnas, mayúsculas en relación a filas (días después de cosecha) con la prueba de Tukey al 0,05. 
Los frutos tratados al momento de la cosecha no presentaron diferencias en firmeza 1 DDC; mientras que cuando se trataron 8 días antes del corte, la dosis 0,16 mM RVS generó mayor firmeza para 'Fino de Jete', mientras que en 'Bronceada' 0,016; 0,16 y 1,6 mM RVS provocaron mayor firmeza en relación a $0 \mathrm{mM}$ de RVS. Además, cuando se aplicó RVS 15 DAC, se presentaron resultados contrastantes ya que los frutos de 'Fino de Jete' menos firmes fueron aquellos tratados con 0,016 mM; mientras que en 'Bronceada' no existió diferencia. Conforme la madurez avanzó, se observó de manera consistente que los frutos tratados con 1,6 mM RVS presentaron los valores más altos de firmeza (Tabla 2 y 3 ) en ambos cultivares. A los 15 DDC, independientemente de la fecha de aplicación, los frutos tratados con 1,6 mM RVS mantuvieron una firmeza superior, en relación al control, entre 3,5 y 4,5 veces en 'Fino de Jete' y de 2 a 3,5 veces en
'Bronceada'. Reportes de Jiménez et al. (2005) citan que la aplicación de RVS en frutos de aguacate evitó la pérdida de agua $\mathrm{y}$ con ello los frutos se mantuvieron turgentes, y por lo tanto la firmeza no disminuyó considerablemente.

Van Buren (1986) expone que al ser RVS un polifenol, puede promover la lignificación de la pared celular y, en cuyo caso, se favorece la protección contra agentes bióticos y abióticos (Anterola y Lewis, 2002). Los resultados de este estudio confirman que RVS afecta positivamente la calidad del fruto, al reducir la tasa de ablandamiento hasta $78 \mathrm{y}$ $54 \%$ para chirimoya 'Fino de Jete' y 'Bronceada', respectivamente, al término de un periodo de 15 días de almacenamiento. El uso de este conocimiento podría potencializar el transporte y posterior comercialización a mayores distancias de esta especie y con ello ser un producto más rentable.

\section{Tabla 3}

Firmeza en poscosecha de fruta de chirimoya 'Bronceada' con aplicación de resveratrol antes de cosecha.

\begin{tabular}{|c|c|c|c|c|c|c|c|c|c|c|c|c|c|}
\hline \multirow{2}{*}{$\begin{array}{l}\text { Días antes } \\
\text { de cosecha }\end{array}$} & \multirow{2}{*}{$\begin{array}{l}\text { Resveratrol } \\
\quad(\mathrm{mM})\end{array}$} & \multicolumn{12}{|c|}{$\begin{array}{l}\text { Firmeza de cáscara }(\mathrm{N}) \text { en poscosecha } \\
\text { (días después de cosecha) }\end{array}$} \\
\hline & & \multicolumn{4}{|c|}{1} & \multicolumn{4}{|c|}{7} & \multicolumn{4}{|c|}{15} \\
\hline \multirow{5}{*}{0} & 0 & 243 & \pm & 2 & $\mathrm{aA}$ & 19 & \pm & 1 & $\mathrm{cB}$ & 11 & \pm & 1,0 & $\mathrm{cC}$ \\
\hline & 0,016 & 243 & \pm & 6 & $\mathrm{aA}$ & 26 & \pm & 2 & bcB & 25 & \pm & 2,0 & $\mathrm{bB}$ \\
\hline & 0,16 & 252 & \pm & 7 & $\mathrm{aA}$ & 30 & \pm & 4 & $\mathrm{bB}$ & 15 & \pm & 0,4 & $\mathrm{cB}$ \\
\hline & 1,6 & 258 & \pm & 7 & $\mathrm{aA}$ & 58 & \pm & 1 & $\mathrm{aB}$ & 38 & \pm & 1,5 & $\mathrm{aC}$ \\
\hline & $\bar{F}$ & \multicolumn{4}{|c|}{1,4} & \multicolumn{4}{|c|}{56,8} & \multicolumn{4}{|c|}{76,3} \\
\hline \multirow{5}{*}{8} & 0 & 210 & \pm & 6 & $\mathrm{bA}$ & 41 & \pm & 1 & $\mathrm{bB}$ & 22 & \pm & 1,4 & $\mathrm{cC}$ \\
\hline & 0,016 & 219 & \pm & 4 & $a b A$ & 54 & \pm & 1 & $\mathrm{aB}$ & 28 & \pm & 0,6 & $\mathrm{bC}$ \\
\hline & 0,16 & 234 & \pm & 4 & $\mathrm{aA}$ & 55 & \pm & 1 & $\mathrm{aB}$ & 39 & \pm & 1,0 & $\mathrm{aC}$ \\
\hline & 1,6 & 225 & \pm & 5 & $a b A$ & 56 & \pm & 2 & $\mathrm{aB}$ & 41 & & 1,8 & $\mathrm{aC}$ \\
\hline & $\mathrm{F}$ & \multicolumn{4}{|c|}{4,5} & \multicolumn{4}{|c|}{22,6} & \multicolumn{4}{|c|}{48,1} \\
\hline \multirow{5}{*}{15} & 0 & 223 & \pm & 4 & $\mathrm{aA}$ & 28 & \pm & 4 & $\mathrm{bB}$ & 14 & \pm & 0,6 & $\mathrm{cC}$ \\
\hline & 0,016 & 224 & \pm & 6 & $\mathrm{aA}$ & 47 & \pm & 2 & $\mathrm{aB}$ & 18 & \pm & 0,9 & $\mathrm{cC}$ \\
\hline & 0,16 & 223 & \pm & 6 & $\mathrm{aA}$ & 52 & \pm & 4 & $\mathrm{aB}$ & 25 & \pm & 1,4 & $\mathrm{bC}$ \\
\hline & 1,6 & 223 & \pm & 7 & $\mathrm{aA}$ & 58 & \pm & 1,5 & $\mathrm{aB}$ & 30 & \pm & 0,9 & $\mathrm{aC}$ \\
\hline & $\mathrm{F}$ & \multicolumn{4}{|c|}{0,0} & \multicolumn{4}{|c|}{19,1} & \multicolumn{4}{|c|}{51,4} \\
\hline
\end{tabular}

Los datos son la media de cuatro repeticiones, un fruto por repetición. Literales minúsculas diferentes implican diferencias estadísticas en relación a columnas, mayúsculas en relación a filas (días después de cosecha) con la prueba de Tukey al 0,05 . 
Color de cáscara; cromaticidad (c); matiz (Hue)

Los resultados para croma demostraron que sólo se observaron diferencias estadísticas $(\mathrm{p} \leq 0,001)$ para DDC, no así para el resto de los factores y las posibles interacciones (Tabla 1). Para matiz (Hue), las diferencias se observaron para DDC y DDC*RVS ( $\mathrm{p} \leq 0,001)$ (Tabla 1$)$, en el resto de los factores no se observaron diferencias. La cosecha de chirimoya se realiza cuando el color de la cáscara cambia de verde a verde-amarillo (Accorsi y Mannica, 1994; Pinto, 2005). En este sentido, la significancia reportada tiene que ver más con la madurez per se, que por la aplicación de RVS. Como se sabe, el bronceado de la cáscara de chirimoya se debe a la oxidación enzimática irreversible causada por la polifenol oxidasa (PPO) y la peroxidada (POD) (Prieto et al., 2007), por lo tanto, se considera que en este trabajo RVS no tuvo el efecto de inhibir dichas enzimas.

\section{Azúcares reductores}

En inicio, los frutos de 'Fino de Jete' a 1 DDC, presentaron mayor contenido de azúcares reductores que los frutos de 'Bronceada' (Tabla 4 y 5). En ambos cultivares, a los 7 DDC los azúcares reductores se incrementaron al doble en relación a 1 DDC; y, a los 15 DDC este incremento continuó en menor cantidad, hasta alcanzar valores promedios de 16,5 y $15,5 \%$ del peso fresco, para 'Fino de Jete' y 'Bronceada', respectivamente. En la maduración de chirimoya, Gutiérrez et al. (1994) exponen que existen cambios de almidón a fructosa y glucosa en forma equivalente, y una acumulación transitoria de sacarosa en su madurez comercial, lo cual explica la significancia de este factor para DAC, DDC y sus interacciones. Con la aplicación de 1,6 mM RVS 8 DAC, en los frutos de 'Fino de Jete' a los 15 DDC se observó la única diferencia estadística, con menor cantidad de este metabolito en relación, exclusivamente, al control $(0 \mathrm{mM}$ RVS). Esta diferencia fue de $2 \%$, lo cual implica una menor dulzura por la aplicación de 1,6 mM de RVS y posiblemente sea explicada por un efecto inhibidor o retardante del proceso de maduración de RVS sobre las chirimoyas.

\section{Tabla 4}

Azúcares reductores en poscosecha de fruta de chirimoya 'Fino de Jete' con aplicación de resveratrol en precosecha.

\begin{tabular}{|c|c|c|c|c|c|c|c|c|c|c|c|c|c|}
\hline \multirow{2}{*}{$\begin{array}{l}\text { Días antes } \\
\text { de cosecha }\end{array}$} & \multirow{3}{*}{$\begin{array}{c}\text { Resve- } \\
\text { ratrol } \\
(\mathrm{mM})\end{array}$} & \multicolumn{12}{|c|}{$\begin{array}{l}\text { Contenido de azúcares reductores (\%) } \\
\text { (días después de cosecha) }\end{array}$} \\
\hline & & \multicolumn{4}{|c|}{1} & \multicolumn{4}{|c|}{7} & \multicolumn{4}{|c|}{15} \\
\hline \multirow{5}{*}{0} & & 7,0 & \pm & 0,1 & $\mathrm{aC}$ & 14,4 & \pm & 0,4 & $\mathrm{aB}$ & 16,1 & \pm & 0,3 & $\mathrm{aA}$ \\
\hline & 0,016 & 6,9 & \pm & 0 & $\mathrm{aC}$ & 13,2 & \pm & 0,2 & $\mathrm{aB}$ & 15,2 & \pm & 0,2 & $\mathrm{aA}$ \\
\hline & 0,16 & 6,9 & \pm & 0,1 & $\mathrm{aC}$ & 12,9 & \pm & 0,4 & $\mathrm{aB}$ & 15,5 & \pm & 0,5 & $\mathrm{aA}$ \\
\hline & 1,6 & 7,0 & \pm & 0,2 & $\mathrm{aC}$ & 13,5 & \pm & 0,5 & $\mathrm{aB}$ & 16,1 & \pm & 0,4 & $\mathrm{aA}$ \\
\hline & $\mathrm{F}$ & \multicolumn{4}{|c|}{0,1} & \multicolumn{4}{|c|}{2,6} & \multicolumn{4}{|c|}{1,6} \\
\hline \multirow{5}{*}{8} & 0 & 6,7 & \pm & 0,3 & $\mathrm{aC}$ & 14,7 & \pm & 0,5 & $\mathrm{aB}$ & 18,2 & \pm & 0,3 & $\mathrm{aA}$ \\
\hline & 0,016 & 6,4 & \pm & 0,2 & $\mathrm{aC}$ & 13,9 & \pm & 0,4 & $\mathrm{aB}$ & 16,3 & \pm & 0,5 & $a b A$ \\
\hline & 0,16 & 6,7 & \pm & 0,2 & $\mathrm{aC}$ & 13,9 & \pm & 0,3 & $\mathrm{aB}$ & 17,1 & \pm & 0,5 & $\mathrm{abA}$ \\
\hline & 1,6 & 6,4 & \pm & 0,1 & $\mathrm{aC}$ & 14,9 & \pm & 0,2 & $\mathrm{aB}$ & 16,3 & \pm & 0,3 & $\mathrm{bA}$ \\
\hline & $\mathrm{F}$ & \multicolumn{4}{|c|}{1,0} & \multicolumn{4}{|c|}{2,4} & \multicolumn{4}{|c|}{4,1} \\
\hline \multirow{5}{*}{15} & 0 & 6,1 & \pm & 0,1 & $\mathrm{aC}$ & 13,5 & \pm & 0,2 & $\mathrm{aB}$ & 16,2 & \pm & 0,2 & $\mathrm{aA}$ \\
\hline & 0,016 & 6,0 & \pm & 0,2 & $\mathrm{aC}$ & 13,2 & \pm & 0,2 & $\mathrm{aB}$ & 15,6 & \pm & 0,8 & $\mathrm{aA}$ \\
\hline & 0,16 & 6,0 & \pm & 0,0 & $\mathrm{aC}$ & 14,0 & \pm & 0,2 & $\mathrm{aB}$ & 16,7 & \pm & 0,9 & $\mathrm{aA}$ \\
\hline & 1,6 & 5,8 & \pm & 0,0 & $\mathrm{aB}$ & 14,0 & \pm & 0,2 & $\mathrm{aA}$ & 16,0 & \pm & 0,2 & $\mathrm{aA}$ \\
\hline & $\mathrm{F}$ & \multicolumn{4}{|c|}{1,7} & \multicolumn{4}{|c|}{3,6} & \multicolumn{4}{|c|}{0,6} \\
\hline
\end{tabular}

Los datos son la media de cuatro repeticiones, un fruto por repetición. Literales minúsculas diferentes implican diferencias estadísticas en relación a columnas, mayúsculas en relación a filas con la prueba de Tukey al 0,05. 
En 'Bronceada' fue menor la cantidad de azúcares reductores en los frutos tratados con 1,6 y $0,16 \mathrm{mM}$ de RVS en relación a los otros dos tratamientos, pero solamente 1 DDC y en los frutos tratados 8 DAC (Tabla 4 y 5). Las diferencias genéticas entre los cultivares pueden estar relacionadas con el hecho de que RVS afectó ligeramente la calidad de pulpa solo en 'Fino de Jete'.

\section{Sólidos solubles totales}

Los contenidos de SST presentaron diferencias significativas $(\mathrm{p} \leq 0,001)$ para $\mathrm{CV}$, DAC, DDC pero no para RVS. Además, las interacciones $\mathrm{Cv}^{*} \mathrm{DAC}$, DAC*DDC y Cv*DAC*DDC $(\mathrm{p} \leq 0,001)$ también presentaron diferencias estadísticas, presumiblemente por las características propias de cada cultivar (Tabla 1). Nomura et al. (1997) menciona que el valor de SST se encuentra relacionado a las cantidades de azúcares reductores y ácido ascórbico. En este sentido los frutos de 'Fino de Jete' presentaron mayor contenido de SST que
'Bronceada' confirmando lo observado en azúcares reductores. Debido a que al momento de la cosecha no se tuvo presencia de jugo, no se determinó el contenido de SST 1 DDC; pero de 7 a 15 DDC existió acumulación de estos metabolitos para ambos cultivares (datos no mostrados). A los 7 DDC, los frutos de 'Fino de Jete' tratados 8 DAC con 0,16 mM RVS presentaron menor contenido de SST en relación a los frutos tratados con 1,6 mM RVS. Para este cultivar no existieron diferencias significativas en el resto de los tratamientos a los 7 y 15 DDC. Por otro lado, las chirimoyas 'Bronceada' tratadas con 1,6 mM RVS 8 DAC presentaron menor contenido de SST que el resto de los tratamientos solo a los 15 DDC. Esta diferencia en SST, de 0,7 a 1,3 ${ }^{\circ} \mathrm{B}$, es ligera pero puede implicar un menor grado de maduración, o retraso en dicho proceso por la aplicación de 1,6 mM RVS. En general, el efecto benéfico de este estilbeno fue solo reducir la tasa de pérdida de firmeza, pero no inhibió la formación de SST.

\section{Tabla 5}

Azúcares reductores en poscosecha de fruta de chirimoya 'Bronceada' con aplicación de resveratrol en precosecha.

\begin{tabular}{|c|c|c|c|c|c|c|c|c|c|c|c|c|c|}
\hline \multirow{2}{*}{$\begin{array}{l}\text { Días antes } \\
\text { de cosecha }\end{array}$} & \multirow{2}{*}{$\begin{array}{c}\text { Resvera } \\
\text {-trol } \\
(\mathrm{mM}) \\
\end{array}$} & \multicolumn{12}{|c|}{$\begin{array}{l}\text { Contenido de azúcares reductores (\%) } \\
\text { (días después de cosecha) }\end{array}$} \\
\hline & & \multicolumn{4}{|c|}{1} & \multicolumn{4}{|c|}{7} & \multicolumn{4}{|c|}{15} \\
\hline \multirow{5}{*}{0} & 0 & 5,0 & \pm & 0,1 & $\mathrm{aC}$ & 12,7 & \pm & 0,1 & $\mathrm{aB}$ & 15,3 & \pm & 0,1 & $\mathrm{aA}$ \\
\hline & 0,016 & 4,9 & \pm & 0,1 & $\mathrm{aC}$ & 12,3 & \pm & 0,3 & $\mathrm{aB}$ & 15,0 & \pm & 0,1 & $\mathrm{aA}$ \\
\hline & 0,16 & 4,9 & \pm & 0,1 & $\mathrm{aC}$ & 11,8 & \pm & 0,6 & $\mathrm{aB}$ & 14,6 & \pm & 0,2 & $\mathrm{aA}$ \\
\hline & 1,6 & 4,8 & \pm & 0 & $\mathrm{aC}$ & 11,4 & \pm & 0,4 & $\mathrm{aB}$ & 15,0 & \pm & 0,3 & $\mathrm{aA}$ \\
\hline & $\mathrm{F}$ & \multicolumn{4}{|c|}{0,6} & \multicolumn{4}{|c|}{2,2} & \multicolumn{4}{|c|}{1,8} \\
\hline \multirow{5}{*}{8} & 0 & 5,9 & \pm & 0,1 & $\mathrm{aC}$ & 13,7 & \pm & 0,3 & $\mathrm{aB}$ & 15,0 & \pm & 0,0 & $\mathrm{aA}$ \\
\hline & 0,016 & 5,3 & \pm & 0,3 & $\mathrm{abC}$ & 14,0 & \pm & 0,4 & $\mathrm{aB}$ & 15,2 & \pm & 0,2 & $\mathrm{aA}$ \\
\hline & 0,16 & 4,9 & \pm & 0,1 & $\mathrm{bC}$ & 13,7 & \pm & 0,6 & $\mathrm{aB}$ & 15,1 & \pm & 0,1 & $\mathrm{aA}$ \\
\hline & 1,6 & 5,0 & \pm & 0,1 & $\mathrm{bC}$ & 13,1 & \pm & 0,7 & $\mathrm{aB}$ & 15,1 & \pm & 0,1 & $\mathrm{aA}$ \\
\hline & $\mathrm{F}$ & \multicolumn{4}{|c|}{9,4} & \multicolumn{4}{|c|}{0,6} & \multicolumn{4}{|c|}{0,2} \\
\hline \multirow{5}{*}{15} & 0 & 5,9 & \pm & 0,1 & $\overline{\mathrm{aC}}$ & 12,6 & \pm & 0,4 & $\mathrm{aB}$ & 16,4 & \pm & 0,2 & $\mathrm{aA}$ \\
\hline & 0,016 & 5,7 & \pm & 0,1 & $\mathrm{aC}$ & 12,3 & \pm & 0,2 & $\mathrm{aB}$ & 16,7 & \pm & 0,2 & $\mathrm{aA}$ \\
\hline & 0,16 & 5,8 & \pm & 0,0 & $\mathrm{aC}$ & 12,5 & \pm & 0,1 & $\mathrm{aB}$ & 16,5 & \pm & 0,5 & $\mathrm{aA}$ \\
\hline & 1,6 & 5,8 & \pm & 0,0 & $\mathrm{aC}$ & 12,2 & \pm & 0,3 & $\mathrm{aB}$ & 16,6 & \pm & 0,2 & $\mathrm{aA}$ \\
\hline & $\mathrm{F}$ & \multicolumn{4}{|c|}{2,3} & \multicolumn{4}{|c|}{0,4} & \multicolumn{4}{|c|}{0,2} \\
\hline
\end{tabular}

Los datos son la media de cuatro repeticiones, un fruto por repetición. Literales minúsculas diferentes implican diferencias estadísticas en relación a columnas, mayúsculas en relación a filas con la prueba de Tukey al 0,05. 


\section{Ácido ascórbico}

Palma et al. (1993) mencionan que en frutos climatéricos como chirimoya, el contenido de ácidos orgánicos disminuye al evolucionar la maduración. Lo anterior se observó en este trabajo ya que, en promedio, los frutos de 'Fino de Jete' se cortaron con $293 \mathrm{mg} \mathrm{kg}$ de ácido ascórbico (AA) y 15 DDC presentaron 141 $\mathrm{mg} \mathrm{kg}^{-1}$. De la misma manera, la disminución de AA fue de 294 a 165 mg kg-1 en chirimoya 'Bronceada' (datos no mostrados). El ANOVA muestra que RVS no tuvo efecto para AA, y en cada cultivar, solo se observaron diferencias en 'Fino de Jete' 15 DDC con menor AA al aplicar 0,16 mM RVS aplicado al día de la cosecha.

\section{Análisis sensorial}

Debido a que el Anova entre cultivares no mostró diferencias significativas para $\mathrm{Cv}$, DAC, la interacción $\mathrm{Cv}^{*} \mathrm{DAC}$ ni para RVS*DAC, se procedió con el análisis monofactorial para cada cultivar con la aplicación de RVS a 8 y 15 DAC (Tabla 6 y 7). Para ambos cultivares, los panelistas indicaron mejor apariencia, olor y sabor en los frutos con 1,6 mM RVS, al menos con respecto a los frutos control. Sin embargo, en cuanto al color, en 'Fino de Jete' los panelistas calificaron mejor el color de los frutos control en relación a las chirimoyas tratados con 1,6 mM RVS. De manera contraria, en el color para chirimoya 'Bronceada' no se encontró diferencias estadísticas. Cherukuri et al. (2007), mencionan que RVS aportó beneficios en frutos cosechados de mandarina 'Satsuma', al retener el color de la cáscara y el contenido de carotenoides hasta por 8 semanas de almacenamiento. Montero (2000a) aplicó RVS en pimientos 'Lamuyo' y determinó visualmente que RVS mantuvo su color verde 8 días en poscosecha; síntoma de maduración más lenta en comparación de frutos sin tratamiento. En resumen, sensorialmente RVS mantuvo la calidad en chirimoya 'Fino de Jete' y 'Bronceada', esto en cuanto a apariencia, olor y sabor, pero no en color.

\section{Tabla 6}

Evaluación sensorial de chirimoya 'Fino de Jete' almacenada 15 días a temperatura ambiente con previa aplicación de RVS a 8 y 15 días antes de corte

\begin{tabular}{|c|c|c|c|c|c|c|c|c|c|c|c|c|c|}
\hline $\mathrm{RVS}(\mathrm{mM})$ & \multicolumn{4}{|c|}{ Apariencia } & \multicolumn{3}{|c|}{ Color } & \multicolumn{3}{|c|}{ Olor } & \multicolumn{3}{|c|}{ Sabor } \\
\hline 0,0 & 1,56 & \pm & 0,09 & $\mathrm{c}$ & 2,96 & $\pm 0,120$ & $\mathrm{a}$ & 2,30 & $\pm 0,122$ & $\mathrm{~b}$ & 1,78 & $\pm 0,095$ & $\mathrm{c}$ \\
\hline 0,016 & 1,84 & \pm & 0,09 & $\mathrm{c}$ & 2,95 & $\pm 0,356$ & $\mathrm{a}$ & 2,53 & $\pm 0,133$ & $a b$ & 1,92 & $\pm \quad 0,094$ & $\mathrm{c}$ \\
\hline 0,16 & 3,10 & \pm & 0,10 & $\mathrm{~b}$ & 2,31 & $\pm 0,133$ & $a b$ & 2,56 & $\pm 0,140$ & $a b$ & 2,57 & $\pm 0,122$ & b \\
\hline 1,6 & 3,45 & \pm & 0,84 & $\mathrm{a}$ & 2,18 & $\pm 0,116$ & $\mathrm{~b}$ & 2,89 & $\pm 0,155$ & $\mathrm{a}$ & 3,53 & $\pm 0,075$ & $\mathrm{a}$ \\
\hline $\mathrm{F}$ & & 10 & & & & 3,98 & & & 3,09 & & & 65,3 & \\
\hline
\end{tabular}

Los datos son la media de 30 repeticiones, un panelista por repetición. Literales diferentes implican diferencias estadísticas con Tukey $(\mathrm{p}<0,05)$.

\section{Tabla 7}

Evaluación sensorial de chirimoya 'Bronceada' almacenada 15 días a temperatura ambiente con previa aplicación de RVS a 8 y 15 días antes de corte

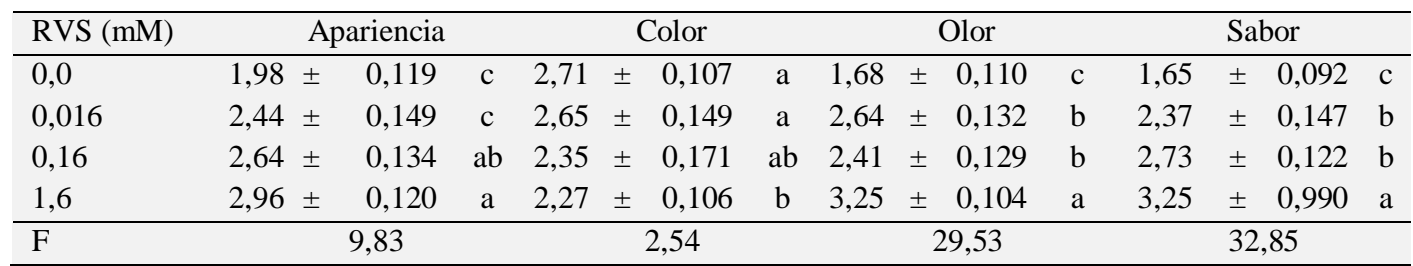

Los datos son la media de 30 repeticiones, un panelista por repetición. Literales diferentes implican diferencias estadísticas con Tukey $(\mathrm{p}<0,05)$. 


\section{Conclusiones}

El resveratrol es un bioregulador vegetal con potencial para mejorar la cadena pos cosecha de chirimoya. Quince días después de almacenados, frutos de chirimoya 'Fino de Jete' y 'Bronceada', tratados 15 días antes de la cosecha con 1,6 mM RVS disminuyeron, respectivamente $\mathrm{y}$ en relación al control, $78 \%$ y $54 \%$ el ablandamiento de la fracción cáscarapulpa. La aplicación de resveratrol no afectó la calidad de la fruta, no se observó diferencia estadística consistente en el periodo de almacenamiento para la tasa de deshidratación y los contenidos de azúcares reductores, sólidos solubles totales y ácido ascórbico. La evaluación sensorial indicó que la aplicación de 1,6 $\mathrm{mM}$ de resveratrol 15 días antes de la cosecha mantuvo mejor la calidad de los frutos en apariencia, olor, textura y sabor.

\section{Agradecimientos}

Esta investigación fue financiada parcialmente por la Universidad Autónoma del Estado de México (proyecto 2678/2008). Se agradece el apoyo de la Fundación Salvador Sánchez Colín-CICTAMEX, especialmente al Ing. Pedro Mijares Oviedo, por permitir trabajar en sus instalaciones. Aaran Aquilino Morales Pérez fue becario de CONACYT.

\section{Referencias bibliográficas}

Accorsi, M. R.; Manica, I. 1994. Colheita, armazenamento e utilizacão. In: fruticultura cultivo das anonáceas: ata cherimólia graviola. Evangraf. Porto Alegre, Brasil.

Anterola, A.; Lewis, N. G. 2002. Trends in lignin modification: a comprehensive analysis of the effects of genetic manipulations mutations on lignification and vascular integrity. Phytochemistry 61: 221-294.

Asif, M. H.; Nath, P. 2005. Expression of multiple forms of polygalacturonase gene during ripening in banana fruit. Plant Physiology \& Biochemistry 43: 177-184.

Cerdas, M. M.; Umana, G.; Castro, J. J. 2006. Manual de manejo poscosecha de anona (Annona cherimola Mill). Ministerio de Agricultura y Ganaderia. San José, Costa Rica.

Committee on Codex Specifications (CCS). 1981. Food chemical codex. National Academy Press. Washigton D. C., USA.

Cherukuri, K.; Woods, F.; Dozier, W.; Ebel, R.; White, D. 2007. Effect of transresveratrol treatment on color retention of satsuma mandarin fruit. Journal of HortScience 42: 982-983.
Ferrucci, P. F. 1997. Estudio global para identificar oportunidades de mercado de frutas y hortalizas de la región andina (primera parte). Informe final. Instituto Interamericano de Cooperación para la AgriculturaAgencia de Cooperación Técnica en Ecuador. Quito, Ecuador.

Gutiérrez, M.; Lahoz, J. M.; Sola, M. D.; Pascual, L.; Vargas, A. M. 1994. Postharvest changes in total soluble solids and tissue $\mathrm{pH}$ of cherimoya fruit stored at chilling and non chilling temperatures. Journal of Horticultural Science 69: 459-463.

Jiménez, J. B.; Orea, J. M.; Montero, C. C.; GonzálezUrena, A.; Navas, E.; Slowing, K.; Gomez- Serranillos, M. P.; Carretero, E.; De Martinis, D. 2005. Resveratrol treatment controls microbial flora, prolongs shelf life, and preserves nutritional quality of fruit. Journal Agricultural and Food Chemistry 53: 1526-1530.

Kabelka, E.; Yang W.; Francis, D. M. 2004. Improved tomato fruit color within an inbred backcross line derived from Lycopersicum esculentum and L. hirsutum involves the interaction of loci. Journal of the American Society for Horticultural Science 129: 250-257.

Lizana, L. A.; Irarrazabal, J. M. 1984. Comportamiento en poscosecha de chirimoya (Anonna cherimolla Mill) sometida a bajas temperaturas y encerado. American Society for Horticultural Science 28: 63-70.

Montero, C.; Bescós, B.; Orea, J. M.; González-Ureña, A. 2000a. Food chemical analysis by laser desorption and resonant ionization mass spectrometry. Reviews in Analytical Chemistry 19: 29.

Montero C.; Orea, J. M.; Muñoz, M. S.; Lobo, R. F.; González-Ureña, A. 2000b. Non volatile analysis in fruits by laser resonant ionization spectrometry: Application to resveratrol in grapes. Journal of Applied Physics 71: 601.

Nomura, K.; Terai, H.; Yabe, K.; Maeda, M.; Rahman, M. S.; Yoshida, M.; Yonemoto, J. Y. 1997. Comparison of changes in sugars and ethylene production of cherimoya fruit grown at different seasons. Journal of Horticultural Science 72: 617-622.

Palma, T., Aguilera, J. M.; Stanley, D. W. 1993. A review of postharvest events in cherimoya. Postharvest Biology and Technology 2: 187-208.

Pedrero, D. L.; Pangborn, R. M. 1997. Evaluación sensorial de los alimentos: Métodos Analíticos. Alhambra Mexicana. Cd. México, México.

Pinto, A. C. Q. 2005. Major and minor production areas. In: Williams, J. T.; Smith, R. W.; Huhes, A.; Haq, N.; Clement, C. R. (eds). Annona Species. International Centre for Underutilized Crops. University of Southampton. Southampton, UK.

Prieto, H.; Utz, D.; Castro, A.; Aguirre, C.; GonzálezAguero, M.; Valdes, H.; Cifuentes, N.; Defilippi, B. G.; Zamora, P.; Zuniga, G.; Campos-Vargas, R. 2007. Browning in Annona cherimola fruit: role of polyphenol oxidase and characterization of a coding sequence of the enzyme. Journal of Agricultural and Food Chemistry 55: 9208-9218.

Ramos, M. G.; Martínez, M. A. 1998. Efecto del manejo poscosecha en la susceptibilidad al daño por frío y la actividad poligalacturonasa en calabaza zuchini. Revista Horticultura Mexicana 6: 42-55.

Valero, D.; Serrano, M. 2010. Postharvest biology and technology for preserving fruit quality. CRC. Boca Raton, USA. 
Van Buren, J. P. 1986. Softening of cooked snap beans and other vegetables in relation to pectins and salts. Chemistry and function of pectins. ACS Symposium Series 310: 190-199.
Zamorano, J.; Alique, R.; Canet, W. 1999. Mechanical parameters to assess quality changes in cherimoya fruit. Zeitschrift für Lebensmittel Untersuchung und Forschung Food Research and Technology 208: 125-129. 